\title{
25\% Albumin Infusion Maintains Antithrombin III (AT) Activity after AT Agent Administration in Critically Ill Patients with Disseminated Intravascular Coagulation (DIC)
}

Mayuki Aibiki $^{1 *}$, Noriyasu Fukuoka ${ }^{2}$, Shiro Bando ${ }^{3}$, Hironori Matsumoto ${ }^{1}$, Muneaki Ohshita ${ }^{1}$, Souichi Maekawam ${ }^{1}$ and Jun Takebe ${ }^{1}$

${ }^{1}$ Department of Emergency Medicine, Ehime University, School of Medicine, Japan

${ }^{2}$ Department of Pharmacology, Nihon University, Japan

${ }^{3}$ Division of Clinical Laboratory, Ehime University Hospital, Japan

\begin{abstract}
Objective: To report for the first time critically ill patients with disseminated intravascular coagulation (DIC) in whom the infusion of $25 \%$ albumin solution produces remarkable elevations in plasma antithrombin III (AT) activities after administration of AT agents.
\end{abstract}

Design: A prospective observational study.

Interventions: 1) Plasma AT activities were serially measured in DIC patients after AT administration, enable us to analyze their pharmacokinetics; 2) Comparisons of AT activities between two groups of receiving AT agent with or without the infusion of $25 \%$ albumin solution; 3 ) in vitro examinations were conducted to define whether albumin application itself would influence directly an AT measurement system using samples already determined the levels of AT activities.

Methods and main results: Twenty consecutive critical patients with DIC were divided into two groups: groups receiving AT agents of 1500 units with $(\mathrm{N}=11)$ and without $(\mathrm{N}=9)$ the application of $25 \%$ albumin solutions. Patients treated with albumin solutions after AT agents showed remarkable elevations in AT peak and trough activities, whereas patients receiving the same dose of the AT agents without albumin co-administration did not maintain its trough activity. Pharmacokinetic analyses revealed that patients had shortened the distribution half-life time of AT, suggesting enhanced vascular permeability. The levels of AT activities in patients treated with both albumin and AT agents $(\mathrm{N}=11)$ were significantly higher than those without albumin administration $(\mathrm{N}=9$, $p=0.01$ ). Furthermore, in vitro albumin applications to the AT measurement system did not effect on its values in the samples.

Conclusion: This is the first report indicating that $25 \%$ albumin administration could elevate and sustain AT activities, even when a limited AT dose was administered to DIC patients with increased vascular permeability. This may be due to a certain binding effect of albumin, which needs to be defined in the future.

Keywords: Albumin; Antithrombin III; Disseminated intravascular coagulation; Pharmacokinetics; Vascular permeability

\section{Introduction}

Antithrombin III (AT) activity beyond its physiological range may obtain anti-coagulatory and anti-inflammatory effects in septic patients with DIC [1]. A large amount of the agent $(6,000$ units for four days) could achieve such goal, but there was a bleeding tendency in such patients with severe platelet depletion [2]. It would be worthwhile if we could maintain effectively AT levels using a certain limited dose of AT agents. We found critical DIC patients in whom 25\% albumin infusion elevated plasma AT activities remarkably after AT agent administration at a dose of 1500 units for three days. After that, we examined retrospectively the effects of $25 \%$ albumin solution after AT administrations on plasma AT activities in critically ill patients with DIC. Furthermore, pharmacokinetic parameters were estimated in such patients.

\section{Subjects and Methods}

General: Twenty consecutive critical patients diagnosed as DIC were reviewed in this study. Patients were divided into two groups: groups receiving AT agents with $(\mathrm{N}=11)$ or without $(\mathrm{N}=9) 25 \%$ albumin solutions (Japan Blood Product Organization: JBPO; CSL Behring Corporation). Demographic data of the patients are shown in the
Table 1. AT agents were administered at 1500 units once per day for three days. Such doses were applied when plasma AT activity decreased below 70\%. This AT dose was used for DIC treatment, which adhered to the recommendation of a government-run Health Care Insurance System in Japan [3]. No co-administration of anti-coagulants such as heparin, gabexate mesilate or nafamostat mesilate was done in any of the patients. Other treatments such as antibiotic administrations, fluid infusion, cardiovascular and respiratory supports were decided by each physician in charge. DIC was diagnosed based on the criteria proposed by the Research Committee on DIC of the Japanese Association for

*Corresponding author: Aibiki M, Department of Emergency and Critical Care Medicine, Ehime University, Graduate School of Medicine, 454 Shitsukawa, Tohon, Ehime, 791-0295, Japan, Tel: +81-89-960-5722; Fax: +81-89-960-5714; E-mail: aibiki@m.ehime-u.ac.jp

Received February 15, 2014; Accepted March 31, 2014; Published April 08 , 2014

Citation: Aibiki M, Fukuoka N, Bando S, Matsumoto H, Ohshita M, et al (2014) 25\% Albumin Infusion Maintains Antithrombin III (AT) Activity after AT Agent Administration in Critically III Patients with Disseminated Intravascular Coagulation (DIC). J Blood Disorders Transf 5: 210. doi: 10.4172/21559864.1000210

Copyright: $\odot 2014$ Aibiki M, et al. This is an open-access article distributed unde the terms of the Creative Commons Attribution License, which permits unrestricted use, distribution, and reproduction in any medium, provided the original author and source are credited. 
Citation: Aibiki M, Fukuoka N, Bando S, Matsumoto H, Ohshita M, et al. (2014) 25\% Albumin Infusion Maintains Antithrombin III (AT) Activity after AT Agent Administration in Critically III Patients with Disseminated Intravascular Coagulation (DIC). J Blood Disorders Transf 5: 208. doi: 10.4172/2155-9864.1000208

Page 2 of 4

\begin{tabular}{|c|c|c|c|c|c|c|c|}
\hline & Age & BW & Gender (F/M) & APACHE II & Pre- ALB (g/dl) & Post-ALB (g/dl) & \\
\hline $\begin{array}{l}\text { AT with ALB } \\
(\mathrm{N}=11)\end{array}$ & $78 \pm 4(73-86)$ & $63 \pm 10(49-67)$ & $4 / 7$ & $30 \pm 4$ & $2.5 \pm 0.7$ & $2.8 \pm 0.5$ & \\
\hline $\begin{array}{l}\text { AT without ALB } \\
(\mathrm{N}=9)\end{array}$ & $75 \pm 7(69-80)$ & $60 \pm 9(50-72)$ & $5 / 4$ & $31 \pm 5$ & $2.6 \pm 0.6$ & $2.7 \pm 0.6$ & \\
\hline \multirow[t]{2}{*}{$P$ values } & 0.86 & 0.3 & 0.5 & 0.2 & 0.26 & 0.3 & \\
\hline & $\begin{array}{c}\text { PLAT. } \\
\text { (XIOOOO/uL) }\end{array}$ & APTT (sec.) & FIB.(mg/dl) & $\operatorname{FDP}(\mathrm{ug} / \mathrm{ml})$ & PT(INR) & CRP (mg/dl) & SEPSIS (+) \\
\hline $\begin{array}{l}\text { AT with ALB } \\
(\mathrm{N}=11)\end{array}$ & $9.1 \pm 3$ & $41 \pm 10$ & $372 \pm 143$ & $25 \pm 15$ & $1.9 \pm 1.2$ & $15.2 \pm 8$ & $9 / 11$ \\
\hline $\begin{array}{l}\text { AT without ALB } \\
(\mathrm{N}=9)\end{array}$ & $91.5 \pm 5$ & $39 \pm 7$ & $365 \pm 182$ & $22 \pm 11$ & $2.0 \pm 1.0$ & $17.1 \pm 10$ & $7 / 9$ \\
\hline$P$ values & 0.4 & 0.24 & 0.43 & 0.5 & 0.65 & 0.56 & 0.55 \\
\hline
\end{tabular}

Pre-ALB: serum albumin levels before AT agent administration with or without $25 \%$ albumin solution; Post-ALB: serum albumin levels after AT agent administration with or without $25 \%$ albumin solution three days after the start of AT agent. The levels of the other parameters are those before the administration of the agent. Data are expressed mean \pm SD. NS: not significant

Table 1: Demographic data of patients.

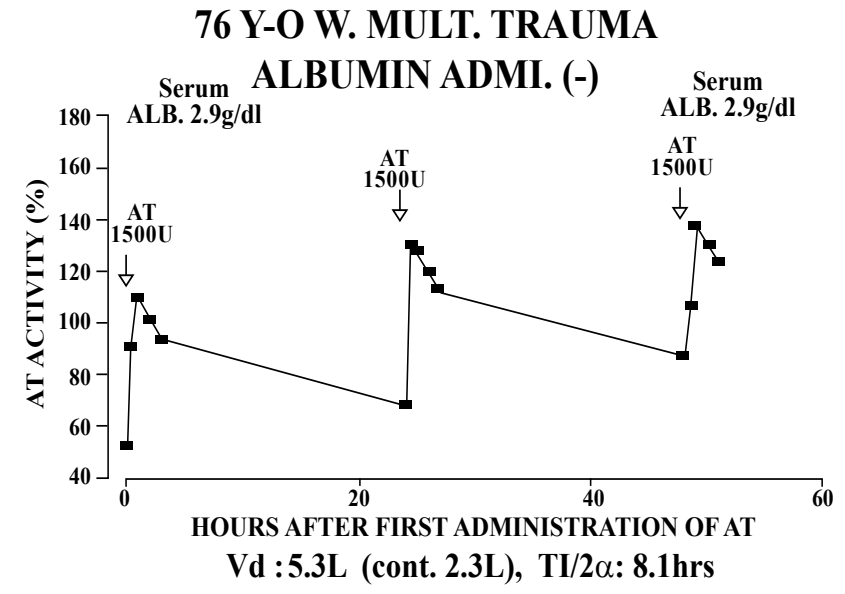

Figure 1A: Depicts a case who showed transient increases in AT activity after AT agent at a dose of 1500 units, which were followed by a return to near the pre-administration levels. MULTI TRAUMA: Multiple Trauma; Vd, distribution volume; T1/2 $\alpha$, half-life on the distribution phase. Cont: Control.

Acute Medicine criteria [4]. Sepsis diagnosis was made according to established criteria [5]. This retrospective study was approved to publish by the Ethics Committee of Ehime University Hospital.

Measurement for AT activity, AT antigen and albumin: Plasma AT antigen and activity were measured by a latex agglutination method (NS auto-AT kit, Naska Corp., Tokyo, Japan; Bio-majesty analyzer, Nihon Denshi Corp. Japan) and a synthesized substrate assay (Test-team S kit for AT, Dai-Ichi Kagaku Corp., Tokyo, Japan; CA 7000 analyzer, Sysmexs, Tokyo, Japan), respectively. Serum albumin levels were determined by the Bromocresol Green method, followed by reflection spectrometry (Auto-analyzer 7450, Hitachi, Japan). The normal range for each test is as follows: AT antigen, $19-30 \mathrm{mg} / \mathrm{dl}$; AT activity, 70-110\%; albumin, 3.5-4.5 g/dl, respectively.

PK analysis for AT: A two-compartment model was used for this study. In all patients, AT activities were measured from plasma samples taken at the pre-infusion (trough level), 30, 60, 120, and 180 minutes after the initiation of the agent. Exceptionally in a case depicted in Figure 1B, additional plasma samples were obtained twelve hours after AT administration. AT of 1500 units was infused for 60 minutes. Such multiple blood samplings had been done for defining PK profile in critical patients with DIC. MULTI [6], a non-linear least square method
76 Y-O W. TBI \& MULT. FRACT.

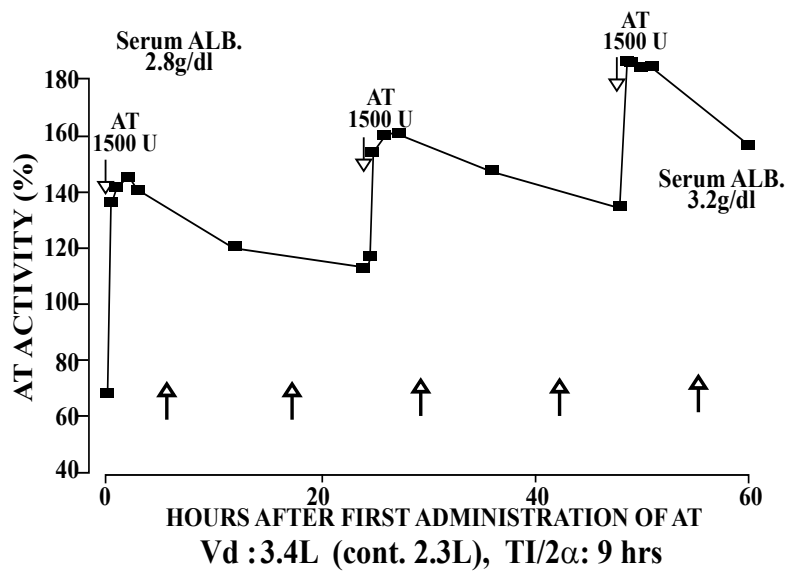

Figure 1B: In contrast, demonstrates a case that showed remarkable elevations in AT activity after AT agent though with the same dose, but this case received $25 \%$ albumin infusion after each AT challenge. Serum levels of albumin were increased, though not remarkably, from 2.8 to $3.2 \mathrm{~g} / \mathrm{dl}$ on the third day. Vd, distribution volume; $\mathrm{T} 1 / 2 \alpha$, half-life on the distribution phase. Cont: Control. MULTI TRAUMA: Multiple Trauma; TBI: Traumatic Brain Injury.

program, was used with an NEC PC-9801 computer (Tokyo, Japan) for the analyses. The Unit/Antigen Ratio (IU/mg) of AT agent used in this study was $6.3 \pm 0.1$ [7]. AT concentrations in all patients here were assessed through activity (\%) as well as antigen concentrations $(\mathrm{mg} / \mathrm{dl})$. AT activities and antigen levels were correlated well $\left(\mathrm{r}^{2}=0.92\right.$, $\mathrm{p}=0.0001$ ). Therefore, subsequent PK evaluations were done by AT activity results. These procedures were reported in detailed in previous papers [8,9]. As a control, we cited data from a previous paper regarding AT pharmacokinetic analysis in patients with congenital AT deficiency [10]. These patients had normal PK profiles of a steady state.

Ex vivo examination for influence on AT measurement system by albumin application in plasma samples: We used sixteen samples in which AT activities had been already measured to clarify whether ex vivo $25 \%$ albumin application (1/10 volume of each sample volume) affected the AT activity values in each. We examined a correlation of calculated values with direct measured values when each sample was diluted to a 9/10 concentration. As shown in Figure 2B, a good 


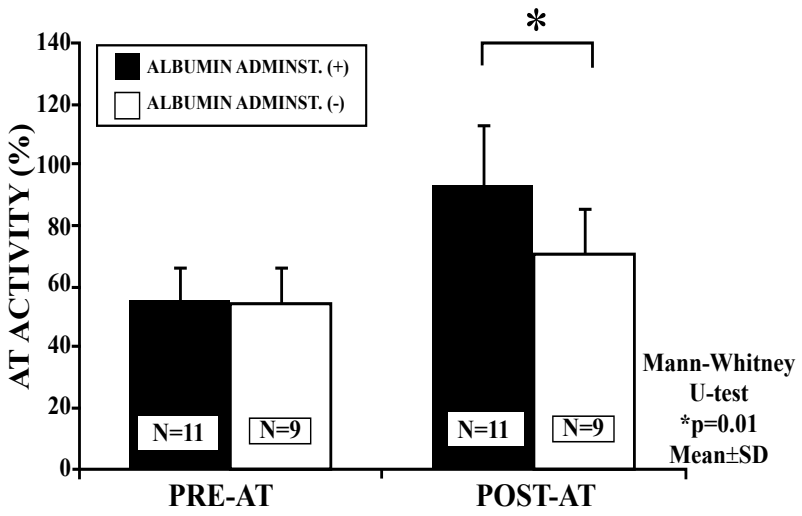

Figure 2A: Shows the comparisons of AT activities between patients with or without albumin administration three days after the start of AT administration.

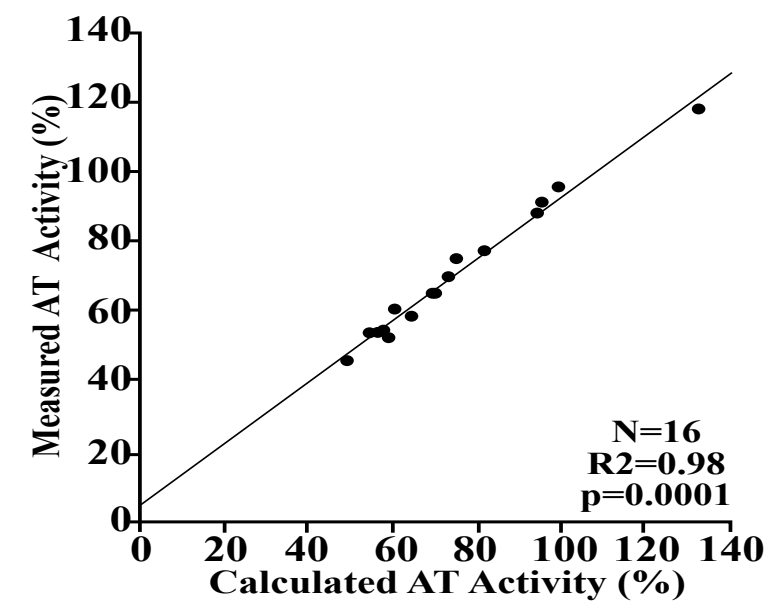

Figure 2B: Demonstrates good correlations between measured and calculated AT activities even after in vitro application of $25 \%$ albumin of $0.5 \mathrm{ml}$

correlation with the two parameters $\left(\mathrm{N}=16, \mathrm{r}^{2}=0.98, \mathrm{p}=0.0001\right)$ was obtained, suggesting that albumin application itself did not significantly affect AT activity values in the measurement system.

\section{Results}

Figure $1 \mathrm{~A}$ is a typical course of a patient receiving only AT agents of 1500 units. The patient showed transient increases in AT activities after AT administration, which were followed by a return to near the pre-administration levels. In contrast, as demonstrated in Figure 1B, a patient who was administered AT agents of 1500 units with $25 \%$ albumin solution showed remarkable elevations in AT activities.

As depicted in Figure 2A, patients receiving AT agents with 25\% albumin solution had a higher level of plasma AT activity than those without albumin administrations three days after the start of AT agent.

Figure $2 \mathrm{~B}$ demonstrates good correlations between measured and calculated AT activities in sixteen samples.

\section{Discussion}

Potential benefits of high concentration albumin on AT activity elevation after $\mathrm{AT}$ agent administration
It would be worth using a certain limited dose of AT agent if we could maintain AT trough levels effectively, since the large amount of the agent should be costly and have a bleeding tendency. There is a previous study for AT agent in septic patients [1], in which two methods were tested: 1) a 6000 unit bolus injection of AT agent followed by an intermittent 1000 unit administration every four hours for four days, 2) the same first bolus infusion followed by continuous AT administration at 250 units/hour for the same period. Both methods sustained AT activities around $150 \%$ even from thirty minutes after the treatment, lasting until the end of study. It is not surprising that such a large dose of AT maintained high levels of AT activity. We have presented here for the first time that in patients with DIC, co-administration of high concentration albumin elevated and sustained AT trough activity around $100 \%$ after AT agent delivered in a dose of just 1500 units once a day for three days. Furthermore, the pharmacokinetic profiles of these patients revealed heightened vascular permeability. Thus, future study is warranted to test the hypothesis that in DIC patients with enhanced vascular permeability, a limited dose of AT improves AT activity when $25 \%$ albumin solutions are co-administered.

\section{Accuracy of AT activity measurement after 25\% albumin application}

We used plasma samples in which AT activities had been already determined to clarify whether in vitro $25 \%$ albumin application $(1 / 10$ volume of each sample volume) affected AT activity values in each sample. We examined a correlation of calculated values with measured values when each sample was diluted to a $9 / 10$ concentration. As shown in Figure 2B, a good correlation with the two parameters $(\mathrm{N}=16$, $\left.\mathrm{r}^{2}=0.98, \mathrm{p}=0.0001\right)$ was obtained, suggesting that albumin application itself did not significantly affect AT activity values in the measurement system. Thus, it appears that the remarkable elevation in AT activity after AT agent with co-administration of $25 \%$ albumin is a biological finding.

\section{Possible mechanisms for AT activity elevation after $25 \%$} albumin administration

Numerous biological effects of high concentration albumin have been reported including its binding capacity to other molecules [11] and attenuation of increased vascular permeability [12]. In this report, we have presented that patients had heightened vascular permeability and augmented distribution volume. Furthermore, we have suggested in a previous paper that reductions in albumin as well as AT in patients with DIC are due mainly to capillary leakage [8]. Therefore, it is likely that the two above-mentioned albumin mechanisms could be a cause for the remarkable elevation presented in this paper in AT activity after administration of AT agent with albumin. However, since the changes in AT activity after albumin infusion occurred so rapidly, it can be speculated that albumin binding capacity rather than attenuation of vascular permeability is more closely related to the present findings. Therefore, we need to test such a hypothesis in the future.

\section{References}

1. Ilias W, List W, Decruyenaere J, Lignian H, Knaub S, et al. (2000) Antithrombin III in patients with severe sepsis: a pharmacokinetic study. Intensive Care Med 26: 704-715.

2. Warren BL, Eid A, Singer P, Pillay SS, Carl P, et al. (2001) Caring for the critically ill patient. High-dose antithrombin III in severe sepsis: a randomized controlled trial. JAMA 286: 1869-1878.

3. Sakata Y, Yoshida N, Matsuda M (1983) Treatment of DIC with Antithrombin III Concentrates. In: Abe T, Yamanaka M (Eds.), Disseminated Intravascular Coagulation. University of Tokyo Press, Tokyo, Japan, pp: 307-316. 
Citation: Aibiki M, Fukuoka N, Bando S, Matsumoto H, Ohshita M, et al. (2014) 25\% Albumin Infusion Maintains Antithrombin III (AT) Activity after AT Agent Administration in Critically III Patients with Disseminated Intravascular Coagulation (DIC). J Blood Disorders Transf 5: 208. doi: 10.4172/2155-9864.1000208

4. Gando S, Saitoh D, Ogura H, Mayumi T, Koseki K (2009) Disseminated intravascular coagulation (DIC) diagnosed based on the Japanese Association for Acute Medicine criteria is a dependent continuum to overt DIC in patients with sepsis. Thromb Res 123: 715-718.

5. Levy MM, Fink MP, Marshall JC, Abraham E, Angus D, et al. (2003) 2001 SCCM/ESICM/ACCP/ATS/SIS International Sepsis Definitions Conference. Crit Care Med 31: 1250-1256.

6. Yamaoka K, Tanigawara Y, Nakagawa T, Uno T (1981) A pharmacokinetic analysis program (multi) for microcomputer. J Pharmacobiodyn 4: 879-885.

7. Gröner A, Nowak T, Römisch J (2002) Purity, activity, and virus safety of a pasteurized antithrombin concentrate. Semin Thromb Hemost 28 Suppl 1: 79-86.

8. Aibiki M, Fukuoka N, Umakoshi K, Ohtsubo S, Kikuchi S (2007) Serum albumin levels anticipate antithrombin III (AT) activities before and after AT agent in critical patients with disseminated intravascular coagulation. Shock 27: 139-144.

9. Aibiki M, Fukuoka N, Nishiyama T, Maekawa S, Shirakawa Y (2007) Differences in antithrombin III activities by administration method in critical patients with disseminated intravascular coagulation: a pharmacokinetic study. Shock 28 : $141-147$.

10. Nakagawa M, Tsuji H, Matsumura H (1985) Changes in post-administered Antithrombin III activity in congenital AT III deficiency patients. Sinryo to Sinyaku 22: $2139-2146$.

11. Dockal M, Carter DC, Rüker F (1999) The three recombinant domains of human serum albumin. Structural characterization and ligand binding properties. J Bio Chem 274: 29303-29310.

12. Victorino GP, Chong TJ, Curran B (2004) Albumin impacts the effects of tonicity on microvascular hydraulic permeability. J Surg Res 122: 167-172.
Citation: Aibiki M, Fukuoka N, Bando S, Matsumoto H, Ohshita M, et al. (2014) 25\% Albumin Infusion Maintains Antithrombin III (AT) Activity after AT Agent Administration in Critically III Patients with Disseminated Intravascular Coagulation (DIC). J Blood Disorders Transf 5: 210. doi: 10.4172/2155 9864.1000210 